\title{
Four-body neutron-recoil recombination reaction as an alternative path for bridging the $A=5,8$ gap in neutron-rich nucleosynthesis scenarios
}

\author{
D.V. Fedorov ${ }^{1, a}$, R. de Diego ${ }^{2}$, E. Garrido ${ }^{2}$, and A.S. Jensen ${ }^{1}$ \\ 1 Department of Physics and Astronomy, University of Aarhus, DK-8000 Aarhus C, Denmark \\ 2 Instituto de Estructura de la Materia, CSIC, Serrano 123, E-28006 Madrid, Spain
}

\begin{abstract}
In neutron-rich nucleosynthesis scenarios the mass gaps $A=5,8$ are assumed to be bridged by threebody electromagnetic recombination reaction $\alpha(\alpha n, \gamma)^{9} \mathrm{Be}$. We suggest an alternative path: the nuclear four-body neutron-recoil recombination reaction $\alpha(\alpha n n, n)^{9} \mathrm{Be}$. A qualitative estimate of the reaction rate for the alternative reaction shows that for higher neutron densities the rate is comparable with the electromagnetic rate.
\end{abstract}

\section{Introduction}

In post-collapse supernovae scenarios of astrophysical nucleosynthesis heavy elements are formed by rapid neutron capture in a hot neutron-rich environment with $10^{20-30}$ neutrons per $\mathrm{cm}^{3}$ and temperatures of the order of $10^{9} \mathrm{~K}[1]$.

The recombination of two $\alpha$-particles and a neutron into ${ }^{9} \mathrm{Be}$ is an important process in these scenarios as it bridges the gaps of unstable isotopes with mass numbers $A=5$ and $A=8$ [2]. The recombination is assumed to proceed through the three-body electromagnetic reaction,

$$
\alpha(\alpha n, \gamma){ }^{9} \mathrm{Be},
$$

where the excess energy is taken away by the emitted photon [2-5].

However in neutron-rich environments the recombination can also occur through the four-body nuclear recombination reaction

$$
\alpha(\alpha n n, n)^{9} \mathrm{Be},
$$

where the excess energy is passed to a neutron in the environment.

Compared to the electromagnetic reaction (1) the production rate of the nuclear process (2) has an extra factor: the density of the available recoil neutrons in the environment. Assuming the typical range of nuclear forces is about $2 \mathrm{fm}$, the characteristic neutron density for the nuclear process is be about $(2 \mathrm{fm})^{-3}=10^{38} \mathrm{~cm}^{-3}$. Consequently, even for the higher estimate of the neutron density in the $r$-process environments, $10^{30} \mathrm{~cm}^{-3}$, the nuclear fourbody recombination (2) has an extra small factor of $10^{-8}$ and therefore was always assumed to be negligible compared to the electromagnetic recombination.

However, simple estimates show that the two processes might be comparable at the higher end of the neutron densities under consideration. Indeed the electromagnetic dipole process involves a factor $(R / \lambda)^{2} \sim 10^{-5}$, where $R \sim$

\footnotetext{
a e-mail: fedorov@phys.au.dk
}

$3 \mathrm{fm}$ is the characteristic size of ${ }^{9} \mathrm{Be}$, and $\lambda=2 \pi \hbar c /(\hbar \omega) \sim$ $800 \mathrm{fm}$, is the wave-length of the emitted photon, where $\hbar \omega \approx 1.6 \mathrm{MeV}$ is the binding energy of ${ }^{9} \mathrm{Be}$. And then there is another small factor, the fine structure constant, $1 / 137$, which makes the total factor $10^{-7}$.

Crucially, the reaction (2) involves low kinetic energies of the particles, as both the temperature, $T \approx 0.1 \mathrm{MeV}$, and the energy released in the reaction, $\approx 1.6 \mathrm{MeV}$, are small on the nuclear scattering scale. The cross-section is thus largely defined by the s-wave scattering lengths between the reacting particles. However the neutron-neutron singlet scattering length, $a_{n n} \approx 20 \mathrm{fm}$, is unusually large on nuclear scale, about an order of magnitude larger than the characteristic range of nuclear forces. Therefore the rate of the nuclear process has an extra large factor, the square of the neutron-neutron scattering length, which gives an extra $10^{2}$

Thus this estimate shows that the rates of electromagnetic and nuclear recombination reactions might be similar at neutron densities of about $10^{29} \mathrm{~cm}^{-3}$.

\section{Neutron-recoil recombination}

We use the participant-spectator model [6], where the transition amplitude $M_{f i}$ for the recombination of the $\alpha \alpha n$ system from an initial (continuum) state $|i\rangle$ to the final (bound) state $\langle f|$ is given as

$$
M_{f i}=\int d^{3} r\left\langle f\left|\frac{e^{-i \mathbf{p}^{\prime} \cdot \mathbf{r}}}{\sqrt{V}} W \frac{e^{+i \mathbf{p} \cdot \mathbf{r}}}{\sqrt{V}}\right| i\right\rangle,
$$

where $\mathbf{r}$ is the relative coordinate between the recoil neutron and the $\alpha \alpha n$ center of mass, $\mathbf{p}$ and $\mathbf{p}^{\prime}$ are the initial and final momenta of the recoil neutron (relative to the $\alpha \alpha n$ system), and $W$ is the interaction between the recoil neutron and the $\alpha \alpha n$ system. The three-body states $|i\rangle$ are normalized to unity within the volume $V$. The final results should be independent of the normalization volume. 
Both the temperature, $10^{9} \mathrm{~K} \sim 0.1 \mathrm{MeV}$, and ${ }^{9} \mathrm{Be}$ binding energy, $\sim 1.6 \mathrm{MeV}$, can be considered as small for the neutron-neutron and neutron- $\alpha$ scattering. In this regime the interaction between the recoil-neutron and the recombining particles is largely determined solely by the corresponding scattering lengths.

The singlet neutron-neutron scattering length, $a_{n n} \approx$ $20 \mathrm{fm}$ is about ten times larger than the neutron- $\alpha$ scattering length. Therefore we can neglect the neutron- $\alpha$ interaction in the matrix element (3) and only leave the neutronneutron interaction.

In the low-energy regime the interaction can be approximated by the Fermi's pseudo-potential particularly suitable for the plane-wave approximation for the recoil neutron,

$$
W=\frac{4 \pi \hbar^{2} a_{n n}}{m} \delta\left(\mathbf{r}_{n n}\right),
$$

where $\mathbf{r}_{n n}$ is the distance between the two neutrons and $m$ is the neutron mass.

Introducing $\mathbf{r}_{n}=\mathbf{r}-\mathbf{r}_{n n}$ as the coordinate between the recombining neutron and the center of mass of the recombining $\alpha \alpha n$ system, and integrating (3) with the $\delta$-function interaction (4) gives

$$
M_{f i}=\frac{4 \pi \hbar^{2} a_{n n}}{V m}\left\langle f\left|e^{-i \mathbf{q} \cdot \mathbf{r}_{n}}\right| i\right\rangle,
$$

where $\mathbf{q}=\mathbf{p}^{\prime}-\mathbf{p}$ is the transferred momentum.

Since the temperature of the environment is smaller than the binding energy of ${ }^{9} \mathrm{Be}$, we neglect the initial thermal energy of the system compared to the recoil energy. Neglecting also the mass of the neutron compared to the mass of ${ }^{9} \mathrm{Be}$ we assume that for any initial state $|i\rangle$ the recoil energy is equal to ${ }^{9} \mathrm{Be}$ binding energy, $\epsilon$. Thus the transferred momentum is given as

$$
q=\sqrt{\frac{2 m \epsilon}{\hbar^{2}}} .
$$

The number of states per unit energy, $d v_{q}$, for the recoil neutron in the final state is given as

$$
d v_{q}=\frac{V d^{3} q}{(2 \pi)^{3}}=\frac{q m V}{(2 \pi)^{3} \hbar^{2}} d \Omega_{q} d E_{q}
$$

where $d \Omega_{q}$ is an infinitesimal solid angle around the direction of $q$ and $E_{q}$ is the energy of the recoil neutron.

The differential probability for the transition $|i\rangle \rightarrow|f\rangle$ for an $\alpha \alpha n$ system per unit time per one recoil neutron is given by the Fermi's Golden rule,

$$
d w_{f i}=\frac{N_{n}}{4} \frac{2 \pi}{\hbar}\left|M_{f i}\right|^{2} \frac{d v_{q}}{d E_{q}},
$$

where $N_{n}$ is the number of available recoil neutrons in the volume $V$, and the factor $1 / 4$ is the probability to find two neutrons in a singlet state in a thermal gas of neutrons.

Substituting the matrix element (5) and the number of states (7) into (8) gives

$$
d w_{f i}=n_{n} a_{n n}^{2} v\left|\left\langle f\left|e^{-i \mathbf{q} \cdot \mathbf{r}_{n}}\right| i\right\rangle\right|^{2} d \Omega_{q},
$$

where $n_{n}$ is the neutron density, $v=\hbar q / m$ is the velocity of the recoil neutron.

Integrating over the recoil angles gives the reaction rate

$$
w_{f i}=n_{n} a_{n n}^{2} v F_{f i}
$$

where

$$
F_{i f}=\int d \Omega_{q}\left|\left\langle f\left|e^{-i \mathbf{q} \cdot \mathbf{r}_{n}}\right| i\right\rangle\right|^{2}
$$

\section{Electromagnetic recombination}

The dipole electromagnetic recombination reaction rate is given as [7]

$$
w_{f i}^{(E 1)}=8 \pi \frac{2}{9} \frac{\omega^{3}}{\hbar c^{3}} B_{f i}^{(E 1)},
$$

where $\hbar \omega$ is the photon's energy, and $B_{f i}^{(E 1)}$ is the reduced dipole transition probability integrated over the photon angles and summed over magnetic quantum numbers of the final state,

$$
B_{f i}^{(E 1)}=4(2 e)^{2} \sum_{\mu \mu_{f}}\left|\left\langle f\left|r_{\alpha} Y_{1 \mu}\left(\mathbf{r}_{\alpha}\right)\right| i\right\rangle\right|^{2},
$$

where $\mathbf{r}_{\alpha}$ is the coordinate of an $\alpha$-particle from center of mass of the recombining $\alpha \alpha n$ system, $2 e$ is the charge of the $\alpha$-particle, $\mu_{f}$ is the magnetic quantum number in the final state of the recombining system, and factor 4 is there because there are two identical $\alpha$-particles.

One can rewrite the rate in a more elucidating form as

$$
w_{f i}^{(E 1)}=\frac{(4 \pi)^{3}}{9} \omega \frac{e^{2}}{\hbar c}\left(\frac{R}{\lambda}\right)^{2} \frac{B_{f i}^{(E 1)}}{e^{2} R^{2}},
$$

where $R^{2} \approx(2 \mathrm{fm})^{2}$ is the mean square charge radius of ${ }^{9} \mathrm{Be}$, and $\lambda$ is the wavelength of the photon.

\section{Qualitative comparison of the nuclear and electromagnetic rates}

The nuclear rate (10) and the electromagnetic rate (14) can be compared qulitatively. Indeed the dimensionless factors $F_{f i}$ and $B_{f i}^{(E 1)} /\left(e^{2} R^{2}\right)$ should be of the same natural order since they contain only quantities of the natural scale of the three-body system. Then the ratio of nuclear to electromagnetic rates can be estimated as

$$
\frac{w_{f i}}{w_{f i}^{(E 1)}} \sim\left(n_{n} a_{n n}^{3}\right) \frac{\left(\frac{v}{c}\right)}{\left(\frac{R}{\lambda}\right)^{2}} \frac{\lambda}{a_{n n}} \sim 10^{5}\left(n_{n} a_{n n}^{3}\right),
$$

where $v / c \sim 0.05$ and $\lambda \sim 800 \mathrm{fm}$. Thus the nuclear rate will be comparable to electromagnetic rate for neutron densities of the order

$$
n_{n} \sim 10^{-5} a_{n n}^{-3} \sim 10^{30} \mathrm{~cm}^{-3},
$$

which is right on the edge of the possible neutron densities. 
$19^{\text {th }}$ International IUPAP Conference on Few-Body Problems in Physics

\section{Conclusion}

We suggest an alternative reaction for bridging the gap of unstable nuclear isotopes with $A=5,8$ in neutron-rich nucleosynthesis scenarios, namely the nuclear four-body recombination reactions $\alpha(\alpha n n, n)^{9} \mathrm{Be}$. We estimate qualitatively the rate of the nuclear recombination within the participant-spectator model and compare it with the rate of the electromagnetic recombination reaction $\alpha(\alpha n, \gamma)^{9} \mathrm{Be}$. We show that for scenarios with the neutron densities of the order of $10^{30} \mathrm{~cm}^{-3}$ the nuclear rate is comparable with the electromagnetic rate.

\section{References}

1. B.S. Meyer G.J. Mathews, W.M. Howard, S.E. Woosley, and R.D. Hoffman, Astrophys. J. 399, 656 (1992).

2. V.D. Efros, H. Oberhummer, A. Pushkin, and I.J. Thompson, Eur. Phys. J. A1 447 (1998).

3. J. Görres, H. Herndl, I.J. Thompson, and M. Wiescher, Phys. Rev. C52, 2231 (1995).

4. A. Bartlett, J. Görres, G.J. Mathews, K. Otsuki, M. Wiescher, D. Frekers, A. Mengoni, and J. Tostevin, Phys. Rev. C74, 015802 (2006).

5. K. Sumiyoshi, H. Utsunomiya, S. Goko, and T. Kajino, Nucl. Phys. A709, 467 (2002).

6. E. Garrido, D.V. Fedorov, and A.S. Jensen, Nucl. Phys. A695, 109 (2001).

7. P.J. Siemens, and A.S. Jensen, Elements of Nuclei, Addison-Wesley, 1987, ISBN 0-201-15572-9 